\title{
Mechanisms of Disease: molecular genetics of arrhythmogenic right ventricular dysplasia/cardiomyopathy
}

\author{
Mark M Awad, Hugh Calkins, and Daniel P Judge* \\ MM Awad is an MD-PhD Candidate at the Johns Hopkins University School of Medicine and the \\ Johns Hopkins University Cellular and Molecular Medicine program, $\mathrm{H}$ Calkins is a Professor of \\ Medicine at the Johns Hopkins University School of Medicine and Director of the \\ Electrophysiology Laboratory at the Johns Hopkins Hospital, and DP Judge is Assistant Professor \\ of Medicine at the Johns Hopkins University School of Medicine and Medical Director of the \\ Center for Inherited Heart Disease at Johns Hopkins Hospital, Baltimore, MD, USA
}

\section{SUMMARY}

Arrhythmogenic right ventricular dysplasia/cardiomyopathy is an inherited cardiomyopathy estimated to affect approximately 1 in 5,000 individuals. Cardinal manifestations include right ventricular enlargement and dysfunction, fibrofatty replacement of myocytes in the right ventricle, characteristic electrocardiographic abnormalities, and ventricular arrhythmia most commonly arising from the right ventricle. The disease is frequently familial and typically involves autosomal dominant transmission with low penetrance and variable expressivity. Approximately $50 \%$ of symptomatic individuals harbor a mutation in one of the five major components of the cardiac desmosome. Nevertheless, other genetic modifiers and environmental factors complicate the clinical management of mutation carriers as well as counseling of their relatives. This Review summarizes the known genetic mutations associated with arrhythmogenic right ventricular dysplasia/cardiomyopathy, describes possible origins of recurrent mutations, presents theories on the pathogenesis of disease following a mutation, and discusses the current issues surrounding clinical use of genetic analysis in the assessment of individuals with this condition.

\section{Keywords}

arrhythmia; arrhythmogenic right ventricular dysplasia; arrhythmogenic right ventricular cardiomyopathy; genetics; sudden cardiac death

\footnotetext{
C2008 Nature Publishing Group

*Correspondence \#1049 Ross Building, 720 Rutland Avenue, Baltimore, MD 21205, USA, djudge@jhmi.edu. REVIEW CRITERIA

This manuscript is based on our experience with ARVD/C and the genetic factors contributing to it, as well as a comprehensive review of published manuscripts on the topic in PubMed. Owing to the many different names for this disorder, we searched using acronyms and full names including "ARVD", "ARVC", "ARVD/C", "ARVC/D", "Uhl's anomaly", and "parchment right ventricle". Historical documents as early as 1952 were used, although the majority of reviewed references were published between 1982 and the present. Review was restricted to full documents, and references cited within documents were used to expand our search.

Medscape Continuing Medical Education online Medscape, LLC is pleased to provide online continuing medical education (CME) for this journal article, allowing clinicians the opportunity to earn CME credit. Medscape, LLC is accredited by the Accreditation Council for Continuing Medical Education (ACCME) to provide CME for physicians. Medscape, LLC designates this educational activity for a maximum of 1.0 AMA PRA Category 1 Credits ${ }^{\mathrm{TM}}$. Physicians should only claim credit commensurate with the extent of their participation in the activity. All other clinicians completing this activity will be issued a certificate of participation. To receive credit, please go to http://www.medscape.com/cme/ncp and complete the post-test.

Competing interests

The authors, the journal editor H Camm and the CME questions author D Lie declared no competing interests.
} 


\section{INTRODUCTION}

Arrhythmogenic right ventricular dysplasia/cardiomyopathy (ARVD/C) is a heritable condition characterized by replacement of cardiomyocytes, primarily in the right ventricle, by fibrofatty tissue. ${ }^{1}$ The resulting disruption of normal myocardial architecture in ARVD/C can result in severe right ventricular (RV) dysfunction, life-threatening arrhythmias and sudden cardiac death. A number of genetic studies have identified mutations in various components of the cardiac desmosome that have important roles in the pathogenesis of ARVD/C (Figure 1). Mutations in ARVD/C-related genes demonstrate incomplete penetrance and variable expressivity, implicating environmental factors and other genetic modifiers in the etiology of this disease. This Review focuses on recent genetic advances, hypothetical disease mechanisms, and the role of clinical genetic testing in diagnosis and prognosis.

In 1952, Henry Uhl described an 8-month-old girl in whom the parietal surface of the RV had become "paper-thin with no myocardium visible" and the endocardium had become apposed to the epicardium. ${ }^{2}$ In the following decades, most cases of right ventricular myocardial dysfunction were classified as 'Uhl's anomaly'. ${ }^{3}$ By the late 1970s, however, French physicians had begun describing a distinct clinical entity, characterized by patchy fibrofatty infiltration of the RV myocardium and with patients usually presenting later in life. ${ }^{4}$ Over the following decade, this clinical entity was recognized as a familial disease with incomplete penetrance and variable expressivity. 5,6

Credit for the original description of ARVD/C is usually granted to a report on 24 cases that focused on the cardiac electrophysiological attributes of the disease. ${ }^{7}$ In addition, the name of this disease state has evolved, from the early eponym of 'Uhl's anomaly' and 'parchment right ventricle', to arrhythmogenic right ventricular dysplasia, which referenced the characteristic histopathology of fibrofatty infiltration of the RV myocardium. A moregeneralized term is now commonly used —arrhythmogenic right ventricular cardiomyopathy -which does not ascribe a primary role to the fibrofatty infiltration. For this report, we use a hybrid eponym, ARVD/C, in an effort to be inclusive.

\section{MUTATIONS}

\section{Plakoglobin}

Studies of individuals from the Greek island of Naxos with an autosomal recessive syndrome characterized by the triad of ARVD/C, non-epidermolytic palmoplantar keratoderma and woolly hair ('Naxos disease') led to identification of the first causative gene for an ARVD/C-associated disorder. Initial mapping of this disorder pointed to the chromosomal locus $17 \mathrm{q} 21,{ }^{8}$ and candidate-gene sequencing within this region revealed a homozygous 2 bp deletion (c.2157-2158delGT) in the junction plakoglobin gene (JUP) that was present only in affected individuals (Figure 2A). ${ }^{9}$ Subsequent studies showed that among subjects homozygous for this mutation, the disease is completely penetrant by adolescence. ${ }^{10} \mathrm{~A}$ study of a German family recently reported the first dominantly inherited JUP mutation (p.Ser39-Lys40insSer) to cause nonsyndromic ARVD/C (i.e. no cutaneous abnormalities). ${ }^{11}$

Plakoglobin, also known as $\gamma$-catenin and a member of the armadillo family of proteins, ${ }^{12}$ was the first component of the desmosome to be implicated in the pathogenesis of ARVD/C. Homozygous targeted disruption of the plakoglobin gene in mice (Jup-/-) results in embryonic lethality, caused by severe heart defects, beginning at embryonic day $10.5 .{ }^{13} \mathrm{In}$ addition to skin blistering, these mice demonstrated ventricular rupture, impaired contractility and an absence of desmosomes in the intercalated discs of the myocardium. ${ }^{13,14}$ 
Mice with heterozygous plakoglobin mutations ( $\mathrm{Jup+/-}$ ) were indistinguishable from their wild-type littermates at birth, but showed RV dilatation and dysfunction, and ventricular arrhythmias by 6 months of age. These phenotypes were exacerbated by exercise (daily swimming for 2 months), ${ }^{15}$ supporting the impression that endurance training could accelerate disease progression among individuals with ARVD/C. Despite these findings, histologic analysis of myocardium from Jup $+/-$ mice did not show any fibrofatty infiltration, and electron microscopy studies revealed no structural changes in the desmosomes or adherens junctions. Furthermore, gene-expression profiling of wild-type and heterozygous mice showed no statistical differences in gene expression patterns. ${ }^{15}$

\section{Desmoplakin}

In the mid-1990s, investigators in India16 and Ecuador ${ }^{17}$ described an autosomal recessive syndrome similar to Naxos disease that was also characterized by woolly hair and palmoplantar keratoderma, but individuals with this syndrome showed predominantly leftsided dilated cardiomyopathy ('Carvajal syndrome'). In the same year that JUP mutations were found in patients with Naxos disease, homozygosity mapping led to the discovery of a desmoplakin gene (DSP) mutation (c.7901delG) in three Ecuadorian families with Carvajal syndrome. ${ }^{18}$ This report was the second to link desmosome dysfunction with a syndrome that included manifestations of ARVD/C. Since this initial study, a number of novel mutations in $D S P$, both recessive and dominant, have been identified in individuals with a constellation of woolly hair, palmoplantar keratoderma and cardiomyopathy. ${ }^{19-21}$ Interestingly, other missense and nonsense mutations in the desmoplakin gene have been associated with isolated dominant ARVD/C or arrhythmogenic left ventricular cardiomyopathy in which affected patients had no hair or skin abnormalities. ${ }^{22-24}$ Furthermore other $D S P$ mutations have been found to cause cutaneous abnormalities without signs of cardiomyopathy. ${ }^{25,26} \mathrm{DSP}$ mutations are summarized in Figure $2 \mathrm{~B}$.

In vivo experiments examining the effects of desmoplakin disruption on heart physiology have provided insight into the pathogenesis of ARVD/C. Desmoplakin, a member of the plakin family, is expressed in all desmosomes, and is a cytoplasmic protein without a transmembrane domain that serves as an intracellular link between desmosomes and intermediate filaments. ${ }^{12,27}$ The desmoplakin mouse knockout ( $\left.D s p-/-\right)$ dies shortly after embryonic implantation at embryonic day 6.5. ${ }^{28}$ Rescue of desmoplakin expression in the extra-embryonic tissues prolongs the survival of $D s p-/-$ mice to embryonic day 10, but these mice display pronounced defects in heart, epidermis and neuroepithelium development. ${ }^{29}$ Cardiac-specific deletion of $D s p$ generates a mouse that exhibits several features of human ARVD/C. ${ }^{30}$ By use of this model, a study found that heterozygous cardiac-tissue-specific $D s p+/-$ mice were normal at birth but displayed a cardiac phenotype at 6 months that included fibrofatty replacement of the right and left ventricular myocardium, thin ventricular walls, impaired left ventricular ejection fraction and ventricular arrhythmias, and premature mortality of $20 \%$.

Another study demonstrated that cardiac-specific overexpression of mutant, but not wildtype, desmoplakin in mice increased myocardial apoptosis, fibrosis and adipose deposition as well as biventricular dilatation and dysfunction. ${ }^{31}$ Although human ARVD/C caused by aberrant $D S P$ overexpression has not been documented, this mouse study indicates that perturbations in the precise ratios of desmosomal components could contribute to disease pathogenesis.

\section{Plakophilin-2}

The discovery of mutations in two functionally related genes, plakoglobin and desmoplakin, focused attention on the desmosome in ARVD/C pathogenesis. Plakophilin-2, an armadillo- 
family member that is expressed in the heart and interacts directly with plakoglobin and desmoplakin, ${ }^{12}$ is essential in mice for proper heart morphogenesis and desmoplakin localization. ${ }^{32}$ Subsequent sequencing of the plakophilin-2 gene (PKP2) in 120 unrelated probands with ARVD/C revealed heterozygous mutations in 32 individuals (a prevalence of $27 \%) .{ }^{33}$ Studies in several other cohorts confirmed that $P K P 2$ mutations in patients with ARVD/C are common, with a prevalence ranging from $11 \%$ to $43 \% .{ }^{34-36}$ Although the vast majority of known mutations are heterozygous and result in missense, nonsense and frameshift mutations, a recessive mutation has been reported, which is notably not associated with hair or skin abnormalities. ${ }^{37}$ These mutations are summarized in Figure 2C. The combination of several reported series in which $P K P 2$ mutation analysis was performed $(n=363)$ resulted in a PKP2 mutation rate of $26 \%$ in unrelated ARVD/C patients. ${ }^{33-36,38}$

The high prevalence of $P K P 2$ mutations has enabled statistical analyses of genotypephenotype correlations in patients with $\mathrm{ARVD} / \mathrm{C}$ as well as of disease penetrance in their relatives. A North American study showed that, in comparison with ARVD/C probands without a $P K P 2$ mutation, those with a $P K P 2$ mutation developed symptoms and arrhythmias at an earlier age, although there was no significant difference in implanted cardioverter-defibrillator firing rates. ${ }^{34}$ By contrast, a report from The Netherlands showed no significant difference in age at initial presentation or incidence of sudden death among family members with and without a $P K P 2$ mutation. ${ }^{36}$ Mutation carriers, however, were more likely than non carriers to have T-wave inversions in the precordial leads on electrocardiography. Differences in these data could be a result of population variations or the small sample sizes in both studies.

Among relatives of individuals previously diagnosed with ARVD/C, van Tintelen et al. found no $P K P 2$ mutations in 11 cases of isolated, non familial ARVD/C. Of 23 patients with well-documented familial ARVD/C, however, $70 \%$ of these probands harbored PKP2 mutations, emphasizing the importance of mutation screening, especially in familial ARVD/ C. ${ }^{36}$ Another study by Dalal and colleagues found that among the PKP2 mutation carrying relatives, $49 \%$ met diagnostic Task Force Criteria for ARVD/C (Table 1). ${ }^{39}$ Of the mutation carriers who did not satisfy diagnostic criteria, 50\% met at least one criterion other than family history. Within families, phenotypic variability was high in individuals carrying the same mutation: some family members were completely asymptomatic in later life, whereas some had severe disease and died prematurely. This study also showed that penetrance of $P K P 2$ mutations was higher with increased age and male sex, with male mutation carriers more likely than female mutation carriers to have both structural and conduction abnormalities. ${ }^{39}$ These data support previous findings among Italian and Western European populations that $\mathrm{ARVD} / \mathrm{C}$ incidence is higher in men than women. ${ }^{40,41}$

The high occurrence of $P K P 2$ mutations among individuals with ARVD/C probably relates to several factors. Although haplotype studies in Dutch patients with matching PKP2 mutations showed inheritance of a common allele, suggesting a founder effect, ${ }^{36}$ microsatellite analysis in larger, more heterogeneous populations in North America ${ }^{34}$ and Western Europe ${ }^{33}$ confirmed inheritance of identical mutations in unrelated alleles. These recurrent mutations suggest that $P K P 2$ contains genomic regions that are inherently prone to alteration. Another possible reason for the raised frequency of $P K P 2$ mutations among different, unrelated families is the presence of the nearby plakophilin-2 pseudo-gene $(P K P 2 P 1)$ located on chromosome $12 \mathrm{p} 13,{ }^{42}$ which could induce $P K P 2$ gene conversion. When compared with the $P K P 2$ coding sequence, the $P K P 2 P 1$ sequence contains a 4-bp deletion corresponding to the c.145-148delCAGA mutation described by several independent groups. ${ }^{33-36}$ However, splice site mutations disrupting critically conserved intronic nucleotides are not likely to be caused by gene conversion, as $P K P 2 P 1$ is a processed pseudogene and, therefore, devoid of introns. An additional potential mechanism 
of recurrent mutations is $\mathrm{C}>\mathrm{T}$ transition at $\mathrm{CpG}$ hotspots; indeed, at least four known $\mathrm{C}>\mathrm{T}$ PKP2 mutations occur at $\mathrm{CpG}$ dinucleotides (c.235C > T, c. 1237C > T, c. 1951C $>\mathrm{T}$ and c. $2203 \mathrm{C}>\mathrm{T})$.

\section{Desmoglein-2}

Desmogleins are desmosomal cadherins, and together with the desmocollins are essential transmembrane components of the desmosome. ${ }^{43}$ There are four related members of the desmoglein family, each with a separate gene localized to chromosome 18p. Desmoglein-2, encoded by $D S G 2$, is highly expressed in cardiac tissue, making it an attractive ARVD/C candidate gene. Sequence analysis led to recognition of $D S G 2$ as the fourth desmosomal gene associated with ARVD/C. ${ }^{44,45}$

To date, the majority of $D S G 2$ mutations identified (summarized in Figure 2D) are extracellular missense mutations. ${ }^{44-46}$ The prevalence of $D S G 2$ mutations in published reports varies from 7-26\%. ${ }^{44,45,47}$ Importantly, the populations analyzed in these studies also varied; some excluded individuals with recognized $P K P 2$ or $D S P$ mutations, ${ }^{44,45}$ and one study included individuals with left ventricular or biventricular cardiomyopathy. ${ }^{47}$ As such, these figures might not represent the true prevalence of $D S G 2$ mutations in cohorts of individuals with ARVD/C.

Individuals with $D S G 2$ mutations-including two probands with compound heterozygous DSG2 mutations and consequently no normal desmoglein-2 - seem to have isolated ARVD/ C without skin or hair abnormalities. ${ }^{44,45}$ By contrast, complete loss of desmoglein-2 in mice is lethal. ${ }^{48}$ Although abnormal desmoglein- 2 expression has been linked with diffusetype gastric cancer in humans, ${ }^{49,50}$ there have been no reports of gastric cancer in patients with ARVD/C carrying DSG2 mutations.

\section{Desmocollin-2}

Desmocollin-2 - another type I integral membrane cadherin found in desmosomes and similar to desmoglein-2 - was the fifth and most recent major component of the cardiac desmosome to be implicated in ARVD/C. ${ }^{51,52}$ Mutations in the desmocollin-2 gene (DSC2) seem to be infrequent in ARVD/C; only five $D S C 2$ mutations have been described to date (summarized in Figure 2E). ${ }^{51-53}$ Although $D s c 2$ targeting in the mouse has not yet been reported, zebrafish treated with $d s c 2$ antisense morpholino oligos demonstrated bradycardia, impaired contractility and chamber dilation. ${ }^{51}$

\section{Non-desmosome gene mutations}

Catecholaminergic polymorphic ventricular tachycardia resembles ARVD/C, though structural right ventricle disease is not typically seen in this disorder. Mutations in RYR2, encoding the cardiac ryanodine receptor, were first reported in 12 individuals with this disorder but no structural RV disease. ${ }^{54}$ Revisiting previous linkage analysis that had shown an association between chromosome 1q42.1-q43 and a phenotype described as ARVD/C then identified mutations in RYR2 in four families. ${ }^{55}$ This particular subtype of ARVD/C had previously been characterized in extensive detail, with a notable paucity of structural $\mathrm{RV}$ disease, but with fibrofatty replacement of right ventricular myocytes on histopathologic examination. ${ }^{56}$ Today, most consider catecholaminergic polymorphic ventricular tachycardia as a distinctly separate disorder from ARVD/C, though interpretation of the clinical criteria used to make these diagnoses can be ambiguous.

An isolated report describes novel variants in the untranslated regions of TGFB3, the gene encoding transforming growth factor $\beta 3$ in an individual with ARVD/C. ${ }^{57}$ This finding raises interesting questions about the role of the pluripotent transforming growth factor $\beta$ 
cytokine family in the pathogenesis of ARVD/C, although a direct causative role has not been proven. Notably, in vitro experiments using these noncoding variants demonstrated twofold increased expression of fusion constructs containing the noncoding variants compared with fusion constructs containing wild-type TGFB3; however, increased expression of $T G F B 3$ in patient-derived tissues was not found, downstream effectors of transforming growth factor $\beta$ signaling were not assessed, and mutational analysis of known desmosomal ARVD/C genes was not reported.

\section{Additional genomic loci}

There are several early reports on linkage analysis in families segregating ARVD/C. ${ }^{58-61}$ Despite extensive investigation within or in close proximity to identified loci, related genes with mutations resulting in true $\mathrm{ARVD} / \mathrm{C}$ have not been reported. One possible reason is that this disorder has both low penetrance and variable expressivity, complicating phenotypic assessment, which is critical for proper assignment of linkage. For example, a family initially reported to show cosegregation of the ARVD/C phenotype with chromosome 2q32.1-q32.3 was subsequently recognized to have a responsible mutation in $P K P 2$, which localizes to chromosome $12 \mathrm{p} 11 .{ }^{33,60}$ At least two family members were classified differently with regard to their phenotype in the later report. ${ }^{33}$

A genomic locus on the short arm of chromosome 3 was initially reported in 1998 in a large REVIEW Newfoundland family segregating ARVD/C. ${ }^{58}$ Subsequently, inclusion of additional individuals and restriction of analysis to only affected individuals refined the locus to $2 \mathrm{cM}$ at chromosome $3 \mathrm{p} 25$ with a logarithm of odds score of $9.3 .{ }^{62}$ Though a specific mutation has not been identified, a haplotype involving this locus has been associated with sudden cardiac death, survival and response to treatment with an implantable cardioverter-defibrillator. ${ }^{62}$ Although eventual discovery of the responsible gene mutation will probably improve our understanding of ARVD/C pathogenesis, clinical assessment of the 3 p25 haplotype in families is certainly justified.

\section{THEORIES ON MECHANISM OF DISEASE}

Several mechanisms have been proposed to explain the association between desmosome gene mutations and RV enlargement and dysfunction, and fibrofatty scar formation (Figure 1). The simplest is a purely structural model that proposes that the loss of myocyte adhesion results in cell death and regional fibrosis. Recent reports, including electron microscopy studies, describe ultrastructural abnormalities of the desmosome associated with desmosome gene mutations, which support this hypothesis. ${ }^{11,38}$ Focal RV scar would then result in the characteristic arrhythmia that typically accompanies ARVD/C. In this structural model, environmental factors such as exercise or inflammation from viral infection could exacerbate impaired adhesion and hasten disease progression. The right ventricle might have greater propensity to disease than the left because of its thinner walls and its normal dilatory response to exercise. The absence of left ventricular disease and the late-onset disease seen in some individuals with clear desmosome gene mutations are not, however, easily explained by this model.

A more complex model invokes the canonical Wnt/ $\beta$-catenin signaling pathway. Plakoglobin ( $\gamma$-catenin), a protein with functional similarities to $\beta$-catenin, can localize both to the plasma membrane and the nucleus. ${ }^{63}$ One recent study demonstrated that disruption of desmoplakin frees plakoglobin from the plasma membrane allowing it to translocate to the nucleus and suppress canonical Wnt/ $\beta$-catenin signaling.30 Wnt signaling can inhibit adipogenesis by preventing mesodermal precursors from differentiating into adipocytes.64 Suppression of Wnt signaling by plakoglobin nuclear localization could, therefore, promote the differentiation of adipose tissue in the cardiac myocardium in patients with ARVD/C.30 
Apoptosis also seems to have a role in the myocardial cell loss seen in ARVD/C. TdTmediated dUTP-biotin nick end-labeling (TUNEL) analysis of myocardial tissue from ARVD/C biopsies demonstrated increased DNA fragmentation that is characteristic of programmed cell death. ${ }^{65}$ Other studies have found increased expression of the apoptotic genes CPP32 (which encodes caspase 3) and BAX (which encodes BCL2-associated X protein) in ARVD/C samples but not in age-matched normal controls. ${ }^{66}, 67$ Cell-line studies have shown that plakoglobin regulates expression of the antiapoptotic gene $B C L 2$ (which encodes B-cell CLL/lymphoma 2 ) and that the $\mathrm{Wnt} / \beta$-catenin signaling pathway modulates the apoptotic response in preadipocytes. ${ }^{68,69}$ Although cardiomyocyte apoptosis seems to be a consistent feature of ARVD/C, the contribution of programmed cell death in the progression and pathogenesis of $\mathrm{ARVD} / \mathrm{C}$ remains unclear.

Elegant functional studies have begun to elucidate the links between mechanical cell junction machinery and electrical gap junction machinery, suggesting a mechanistic link between abnormal desmosomes and arrhythmias. ${ }^{70}$ Interestingly, decreased expression of PKP2 in cardiac cells disrupts the normal localization and conductivity of the gap junction protein connexin $43 .{ }^{71}$ Remodeling of the gap junction in response to an alteration or deficiency in elements of the cardiac desmosome could have a leading role in the genesis of arrhythmias. ${ }^{72}$

Studies of the first-described dominant JUP mutation also shed light on possible mechanisms for ARVD/C pathogenesis. ${ }^{11}$ This in-frame insertional mutation was shown in a yeast-two-hybrid screen to create a novel interaction between mutant plakoglobin and histidine-rich calcium-binding protein. ${ }^{11}$ Although this predicted gain-of-function has not yet been confirmed in patient-derived tissues, these experiments indicate that plakoglobin can promote arrhythmias through aberrant calcium homeostasis mediated by histidine-rich calcium-binding protein. ${ }^{11}$

\section{CURRENT STATUS OF CLINICAL GENETIC ANALYSIS}

Comprehensive exonic sequence analysis of the known desmosomal ARVD/C-related genes currently identifies a responsible mutation in approximately $50 \%$ of ARVD/C probands. Recognition of additional genes associated with this condition, improved techniques for identifying large deletions or gene rearrangements, and lower cost sequence analysis should all improve the diagnostic yield of genetic testing in the future.

Patients and their physicians may seek clinical genetic testing for ARVD/C for several reasons. In our experience, the most common reason cited is identification of individuals related to someone with ARVD/C who may be at increased risk of sudden cardiac death or of developing the disorder. In such cases, the affected proband should be tested first and if a mutation is identified, at-risk family members can also seek testing. Genetic diagnosis before implantation is possible, but depends on the policies in the clinical laboratory performing the test. We discourage testing requested simply on the basis of curiosity or to confirm a diagnosis, as imaging and arrhythmia testing have greater diagnostic utility at this point. When the diagnostic criteria for this disorder are revised, inclusion of desmosome gene mutations in establishing this diagnosis might lead to increased ARVD/C genetic testing in the clinic.

Clinical genetic testing for ARVD/C is currently available in at least two laboratories in the US, both of which meet the Clinical Laboratory Improvement Amendments: the Johns Hopkins DNA Diagnostic Laboratory and the Harvard Laboratory for Molecular Medicine. Clinicians and patients who seek such testing should be aware of the possible outcomes, including the distinct possibility of finding a sequence variant of uncertain or unknown significance in one or more of these genes. Accordingly, genetic counseling is essential with 
such testing. Mutations resulting in insertions, deletions, frameshift, or premature termination are frequently found in this disorder, each of which dramatically alters the predicted protein. Even if they have not been previously reported, mutations may be more easily inferred as pathogenic by a clinical laboratory. Missense substitutions can be harder to interpret without extensive analysis in unaffected populations and functional assessment of these alterations (Figure 3). Clinical genetic testing laboratories are not typically able to perform such analyses.

In our experience, $P K P 2$ mutations are not typically found among individuals with subclinical manifestations of ARVD/C, but are far more common in those who satisfy the current clinical criteria. ${ }^{34}$ As such, use of such clinical genetic testing in an individual who does not meet the diagnostic criteria for ARVD/C is unlikely to result in a clear diagnosis. ${ }^{34}$ As the criteria used in the diagnosis of this condition evolve, genetic testing is likely to provide information that is additive to that provided by family history of ARVD/C.

Several reports have demonstrated probands and families with ARVD/C in whom more than one pathogenic mutation has been identified. $37,44,45$ This possibility must also be considered when advising a proband or family member about the likelihood of identifying those in the family who are at highest risk of developing ARVD/C. As previously noted, penetrance of ARVD/C is low and variable expressivity is widely seen. ${ }^{39}$ Accordingly, identification of a genetic predisposition to ARVD/C should be viewed as only one factor contributing to ARVD/C, and does not independently lead to a diagnosis.

\section{CONCLUSIONS}

$\mathrm{ARVD} / \mathrm{C}$ is a disorder of the cardiac desmosome. Recognition of several genes with mutations contributing to $\mathrm{ARVD} / \mathrm{C}$ has improved our understanding of the pathogenesis of this condition, but importantly also provides an opportunity to effectively target screening within families. New insights derived from cellular and animal studies of the cardiac desmosome are anticipated to improve both diagnosis of and therapy for this condition in the future.

\section{Learning objectives}

Upon completion of this activity, participants should be able to:

1. Describe the prevalence of arrhythmogenic right ventricular dysplasia/ cardiomyopathy (ARVD/C).

2. Identify clinical features of ARVD/C.

3. Describe the histopathology that characterizes ARVD/C.

4. Describe differences between Naxos disease and Carvajal syndrome.

5. Describe optimal screening intervals for family members of patients with ARVD/C.

\section{KEY POINTS}

- Mutation in genes encoding any of the five major components of the cardiac desmosome-PKP2 (encoding plakophilin-2), DSG2 (encoding desmoglein-2), DSP (encoding desmoplakin), DSC2 (encoding desmocollin-2), and JUP (encoding junctional plakoglobin)—can result in arrhythmogenic right ventricular dysplasia/cardiomyopathy (ARVD/C) 
- Approximately 50\% of individuals with ARVD/C who have undergone full sequence analysis of these desmosome genes have a single heterozygous mutation identified, though a few cases of individuals with homozygous or compound heterozygous mutations have also been described

- $\quad \mathrm{ARVD} / \mathrm{C}$ segregates in families with both incomplete penetrance and variable expressivity; clinical screening of family members is recommended, particularly among those recognized to share a genetic predisposition to ARVD/C

- Owing to the age-dependent onset of ARVD/C, repeat clinical screening is recommended at 2- to 3-year intervals from the age of 12 years in the absence of a known mutation, to help target family members at highest risk; in families with earlier onset disease or sudden cardiac death in children, earlier clinical screening should be performed

- With the recent emergence of clinical genetic testing for ARVD/C, genetic counseling is strongly advised for individuals with ARVD/C and their family members

\section{Acknowledgments}

The authors wish to acknowledge funding from the National Institutes of Health (HL088072 to DPJ) and the France-Merrick Foundation. We would also like to acknowledge the Johns Hopkins ARVD Program (www.arvd.com) which is supported by the Bogle Foundation, the Campanella family, and the Wilmerding Endowments. Désirée Lie, University of California, Irvine, CA, is the author of and is solely responsible for the content of the learning objectives, questions and answers of the Medscape-accredited continuing medical education activity associated with this article.

\section{References}

1. McKenna WJ, et al. Diagnosis of arrhythmogenic right ventricular dysplasia/cardiomyopathy. Task Force of the Working Group Myocardial and Pericardial Disease of the European Society of Cardiology and of the Scientific Council on Cardiomyopathies of the International Society and Federation of Cardiology. Br Heart J 1994;71:215-218. [PubMed: 8142187]

2. Uhl HS. A previously undescribed congenital malformation of the heart: almost total absence of the myocardium of the right ventricle. Bull Johns Hopkins Hosp 1952;91:197-209. [PubMed: 12978573]

3. Gerlis LM, et al. Dysplastic conditions of the right ventricular myocardium: Uhl's anomaly vs arrhythmogenic right ventricular dysplasia. Br Heart J 1993;69:142-150. [PubMed: 8435240]

4. Frank R, et al. Electrocardiology of 4 cases of right ventricular dysplasia inducing arrhythmia [French]. Arch Mal Coeur Vaiss 1978;71:963-972. [PubMed: 102297]

5. Nava A, et al. A polymorphic form of familial arrhythmogenic right ventricular dysplasia. Am J Cardiol 1987;59:1405-1409. [PubMed: 3591698]

6. Nava A, et al. Familial occurrence of right ventricular dysplasia: a study involving nine families. J Am Coll Cardiol 1988;12:1222-1228. [PubMed: 3170963]

7. Marcus FI, et al. Right ventricular dysplasia: a report of 24 adult cases. Circulation 1982;65:384398. [PubMed: 7053899]

8. Coonar AS, et al. Gene for arrhythmogenic right ventricular cardiomyopathy with diffuse nonepidermolytic palmoplantar keratoderma and woolly hair (Naxos disease) maps to 17q21. Circulation 1998;97:2049-2058. [PubMed: 9610536]

9. McKoy G, et al. Identification of a deletion in plakoglobin in arrhythmogenic right ventricular cardiomyopathy with palmoplantar keratoderma and woolly hair (Naxos disease). Lancet 2000;355:2119-2124. [PubMed: 10902626]

10. Protonotarios N, et al. Genotype-phenotype assessment in autosomal recessive arrhythmogenic right ventricular cardiomyopathy (Naxos disease) caused by a deletion in plakoglobin. J Am Coll Cardiol 2001;38:1477-1484. [PubMed: 11691526] 
11. Asimaki A, et al. A novel dominant mutation in plakoglobin causes arrhythmogenic right ventricular cardiomyopathy. Am J Hum Genet 2007;81:964-973. [PubMed: 17924338]

12. Getsios S, et al. Working out the strength and flexibility of desmosomes. Nat Rev Mol Cell Biol 2004;5:271-281. [PubMed: 15071552]

13. Bierkamp C, et al. Embryonic heart and skin defects in mice lacking plakoglobin. Dev Biol 1996;180:780-785. [PubMed: 8954745]

14. Ruiz P, et al. Targeted mutation of plakoglobin in mice reveals essential functions of desmosomes in the embryonic heart. J Cell Biol 1996;135:215-225. [PubMed: 8858175]

15. Kirchhof $\mathrm{P}$, et al. Age- and training-dependent development of arrhythmogenic right ventricular cardiomyopathy in heterozygous plakoglobin-deficient mice. Circulation 2006;114:1799-1806. [PubMed: 17030684]

16. Rao BH, et al. Familial occurrence of a rare combination of dilated cardiomyopathy with palmoplantar keratoderma and curly hair. Indian Heart J 1996;48:161-162. [PubMed: 8682558]

17. Carvajal-Huerta L. Epidermolytic palmoplantar keratoderma with woolly hair and dilated cardiomyopathy. J Am Acad Dermatol 1998;39:418-421. [PubMed: 9738775]

18. Norgett EE, et al. Recessive mutation in desmoplakin disrupts desmoplakin-intermediate filament interactions and causes dilated cardiomyopathy, woolly hair and keratoderma. Hum Mol Genet 2000;9:2761-2766. [PubMed: 11063735]

19. Alcalai R, et al. A recessive mutation in desmoplakin causes arrhythmogenic right ventricular dysplasia, skin disorder, and woolly hair. J Am Coll Cardiol 2003;42:319-327. [PubMed: 12875771]

20. Norgett EE, et al. Early death from cardiomyopathy in a family with autosomal dominant striate palmoplantar keratoderma and woolly hair associated with a novel insertion mutation in desmoplakin. 2006;126:1651-1654.

21. Uzumcu A, et al. Loss of desmoplakin isoform I causes early onset cardiomyopathy and heart failure in a Naxos-like syndrome. J Med Genet 2006;43:e5. [PubMed: 16467215]

22. Bauce B, et al. Clinical profile of four families with arrhythmogenic right ventricular cardiomyopathy caused by dominant desmoplakin mutations. Eur Heart J 2005;26:1666-1675. [PubMed: 15941723]

23. Rampazzo A, et al. Mutation in human desmoplakin domain binding to plakoglobin causes a dominant form of arrhythmogenic right ventricular cardiomyopathy. Am J Hum Genet 2002;71:1200-1206. [PubMed: 12373648]

24. Norman M, et al. Novel mutation in desmoplakin causes arrhythmogenic left ventricular cardiomyopathy. Circulation 2005;112:636-642. [PubMed: 16061754]

25. Jonkman MF, et al. Loss of desmoplakin tail causes lethal acantholytic epidermolysis bullosa. Am J Hum Genet 2005;77:653-660. [PubMed: 16175511]

26. Whittock NV, et al. Compound heterozygosity for non-sense and mis-sense mutations in desmoplakin underlies skin fragility/woolly hair syndrome. J Invest Dermatol 2002;118:232-238. [PubMed: 11841538]

27. Smith EA, Fuchs E. Defining the interactions between intermediate filaments and desmosomes. J Cell Biol 1998;141:1229-1241. [PubMed: 9606214]

28. Gallicano GI, et al. Desmoplakin is required early in development for assembly of desmosomes and cytoskeletal linkage. J Cell Biol 1998;143:2009-2022. [PubMed: 9864371]

29. Gallicano GI, et al. Rescuing desmoplakin function in extra-embryonic ectoderm reveals the importance of this protein in embryonic heart, neuroepithelium, skin and vasculature.

Development 2001;128:929-941. [PubMed: 11222147]

30. Garcia-Gras E, et al. Suppression of canonical Wnt/beta-catenin signaling by nuclear plakoglobin recapitulates phenotype of arrhythmogenic right ventricular cardiomyopathy. J Clin Invest 2006;116:2012-2021. [PubMed: 16823493]

31. Yang Z, et al. Desmosomal dysfunction due to mutations in desmoplakin causes arrhythmogenic right ventricular dysplasia/cardiomyopathy. Circ Res 2006;99:646-655. [PubMed: 16917092]

32. Grossmann KS, et al. Requirement of plakophilin 2 for heart morphogenesis and cardiac junction formation. J Cell Biol 2004;167:149-160. [PubMed: 15479741] 
33. Gerull B, et al. Mutations in the desmosomal protein plakophilin-2 are common in arrhythmogenic right ventricular cardiomyopathy. Nat Genet 2004;36:1162-1164. [PubMed: 15489853]

34. Dalal D, et al. Clinical features of arrhythmogenic right ventricular dysplasia/cardiomyopathy associated with mutations in plakophilin-2. Circulation 2006;113:1641-1649. [PubMed: 16549640]

35. Syrris $\mathrm{P}$, et al. Clinical expression of plakophilin-2 mutations in familial arrhythmogenic right ventricular cardiomyopathy. Circulation 2006;113:356-364. [PubMed: 16415378]

36. van Tintelen JP, et al. Plakophilin-2 mutations are the major determinant of familial arrhythmogenic right ventricular dysplasia/cardiomyopathy. Circulation 2006;113:1650-1658. [PubMed: 16567567]

37. Awad MM, et al. Recessive arrhythmogenic right ventricular dysplasia due to novel cryptic splice mutation in PKP2. Hum Mutat 2006;27:1157. [PubMed: 17041889]

38. Lahtinen AM, et al. Plakophilin-2 missense mutations in arrhythmogenic right ventricular cardiomyopathy. Int J Cardiol. 2007 [doi:10.1016/j.ijcard.2007.03.137].

39. Dalal D, et al. Penetrance of mutations in plakophilin-2 among families with arrhythmogenic right ventricular dysplasia/cardiomyopathy. J Am Coll Cardiol 2006;48:1416-1424. [PubMed: 17010805]

40. Nava A, et al. Clinical profile and long-term followup of 37 families with arrhythmogenic right ventricular cardiomyopathy. J Am Coll Cardiol 2000;36:2226-2233. [PubMed: 11127465]

41. Hamid MS, et al. Prospective evaluation of relatives for familial arrhythmogenic right ventricular cardiomyopathy/dysplasia reveals a need to broaden diagnostic criteria. J Am Coll Cardiol 2002;40:1445-1450. [PubMed: 12392835]

42. Bonne S, et al. Assignment of the plakophilin-2 gene (PKP2) and a plakophilin-2 pseudogene (PKP2P1) to human chromosome bands 12p11 and 12p13, respectively, by in situ hybridization. Cytogenet Cell Genet 2000;88:286-287. [PubMed: 10828611]

43. Schwarz MA, et al. Desmosomes and hemidesmosomes: constitutive molecular components. Annu Rev Cell Biol 1990;6:461-491. [PubMed: 2275820]

44. Awad MM, et al. DSG2 mutations contribute to arrhythmogenic right ventricular dysplasia/ cardiomyopathy. Am J Hum Genet 2006;79:136-142. [PubMed: 16773573]

45. Pilichou K, et al. Mutations in desmoglein-2 gene are associated with arrhythmogenic right ventricular cardiomyopathy. Circulation 2006;113:1171-1179. [PubMed: 16505173]

46. Syrris P, et al. Desmoglein-2 mutations in arrhythmogenic right ventricular cardiomyopathy: a genotype-phenotype characterization of familial disease. Eur Heart J 2007;28:581-588. [PubMed: 17105751]

47. Sen-Chowdhry S, et al. Clinical and genetic characterization of families with arrhythmogenic right ventricular dysplasia/cardiomyopathy provides novel insights into patterns of disease expression. Circulation 2007;115:1710-1720. [PubMed: 17372169]

48. Eshkind L, et al. Loss of desmoglein 2 suggests essential functions for early embryonic development and proliferation of embryonal stem cells. Eur J Cell Biol 2002;81:592-598. [PubMed: 12494996]

49. Biedermann K, et al. Desmoglein 2 is expressed abnormally rather than mutated in familial and sporadic gastric cancer. J Pathol 2005;207:199-206. [PubMed: 16025435]

50. Yashiro M, et al. Decreased expression of the adhesion molecule desmoglein-2 is associated with diffuse-type gastric carcinoma. Eur J Cancer 2006;42:2397-2403. [PubMed: 16890424]

51. Heuser A, et al. Mutant desmocollin-2 causes arrhythmogenic right ventricular cardiomyopathy. Am J Hum Genet 2006;79:1081-1088. [PubMed: 17186466]

52. Syrris P, et al. Arrhythmogenic right ventricular dysplasia/cardiomyopathy associated with mutations in the desmosomal gene desmocollin-2. Am J Hum Genet 2006;79:978-984. [PubMed: 17033975]

53. Beffagna G, et al. Missense mutations in desmocollin-2 N-terminus, associated with arrhythmogenic right ventricular cardiomyopathy, affect intracellular localization of desmocollin-2 in vitro. BMC Med Genet 2007;8:65. [PubMed: 17963498] 
54. Priori SG, et al. Mutations in the cardiac ryanodine receptor gene (hRyR2) underlie catecholaminergic polymorphic ventricular tachycardia. Circulation 2001;103:196-200. [PubMed: 11208676]

55. Tiso $\mathrm{N}$, et al. Identification of mutations in the cardiac ryanodine receptor gene in families affected with arrhythmogenic right ventricular cardiomyopathy type 2 (ARVD2). Hum Mol Genet 2001;10:189-194. [PubMed: 11159936]

56. Rampazzo A, et al. A new locus for arrhythmogenic right ventricular cardiomyopathy (ARVD2) maps to chromosome 1q42-q43. Hum Mol Genet 1995;4:2151-2154. [PubMed: 8589694]

57. Beffagna $\mathrm{G}$, et al. Regulatory mutations in transforming growth factor-beta3 gene cause arrhythmogenic right ventricular cardiomyopathy type 1 . Cardiovasc Res 2005;65:366-373. [PubMed: 15639475]

58. Ahmad F, et al. Localization of a gene responsible for arrhythmogenic right ventricular dysplasia to chromosome 3p23. Circulation 1998;98:2791-2795. [PubMed: 9860777]

59. Li D, et al. The locus of a novel gene responsible for arrhythmogenic right-ventricular dysplasia characterized by early onset and high penetrance maps to chromosome 10p12-p14. Am J Hum Genet 2000;66:148-156. [PubMed: 10631146]

60. Rampazzo A, et al. ARVD4, a new locus for arrhythmogenic right ventricular cardiomyopathy, maps to chromosome 2 long arm. Genomics 1997;45:259-263. [PubMed: 9344647]

61. Severini GM, et al. A new locus for arrhythmogenic right ventricular dysplasia on the long arm of chromosome 14. Genomics 1996;31:193-200. [PubMed: 8824801]

62. Hodgkinson KA, et al. The impact of implantable cardioverter-defibrillator therapy on survival in autosomal-dominant arrhythmogenic right ventricular cardiomyopathy (ARVD5). J Am Coll Cardiol 2005;45:400-408. [PubMed: 15680719]

63. Simcha I, et al. Differential nuclear translocation and transactivation potential of beta-catenin and plakoglobin. J Cell Biol 1998;141:1433-1448. [PubMed: 9628899]

64. Ross SE, et al. Inhibition of adipogenesis by Wnt signaling. Science 2000;289:950-953. [PubMed: 10937998]

65. Nagata M, et al. Apoptotic cell death in arrhythmogenic right ventricular cardiomyopathy: a comparative study with idiopathic sustained ventricular tachycardia. Jpn Heart J 2000;41:733-741. [PubMed: 11232990]

66. Yamaji K, et al. Apoptotic myocardial cell death in the setting of arrhythmogenic right ventricular cardiomyopathy. Acta Cardiol 2005;60:465-470. [PubMed: 16261775]

67. Mallat Z, et al. Evidence of apoptosis in arrhythmogenic right ventricular dysplasia. N Engl J Med 1996;335:1190-1196. [PubMed: 8815941]

68. Hakimelahi S, et al. Plakoglobin regulates the expression of the anti-apoptotic protein BCL-2. J Biol Chem 2000;275:10905-10911. [PubMed: 10753888]

69. Longo KA, et al. Wnt signaling protects 3T3-L1 preadipocytes from apoptosis through induction of insulinlike growth factors. J Biol Chem 2002;277:38239-38244. [PubMed: 12154096]

70. Shaw RM, et al. Microtubule plus-end-tracking proteins target gap junctions directly from the cell interior to adherens junctions. Cell 2007;128:547-560. [PubMed: 17289573]

71. Oxford EM, et al. Connexin43 remodeling caused by inhibition of plakophilin-2 expression in cardiac cells. Circ Res 2007;101:703-711. [PubMed: 17673670]

72. Saffitz JE, et al. Remodeling of gap junctions in ischemic and nonischemic forms of heart disease. J Membr Biol 2007;218:65-71. [PubMed: 17585361] 


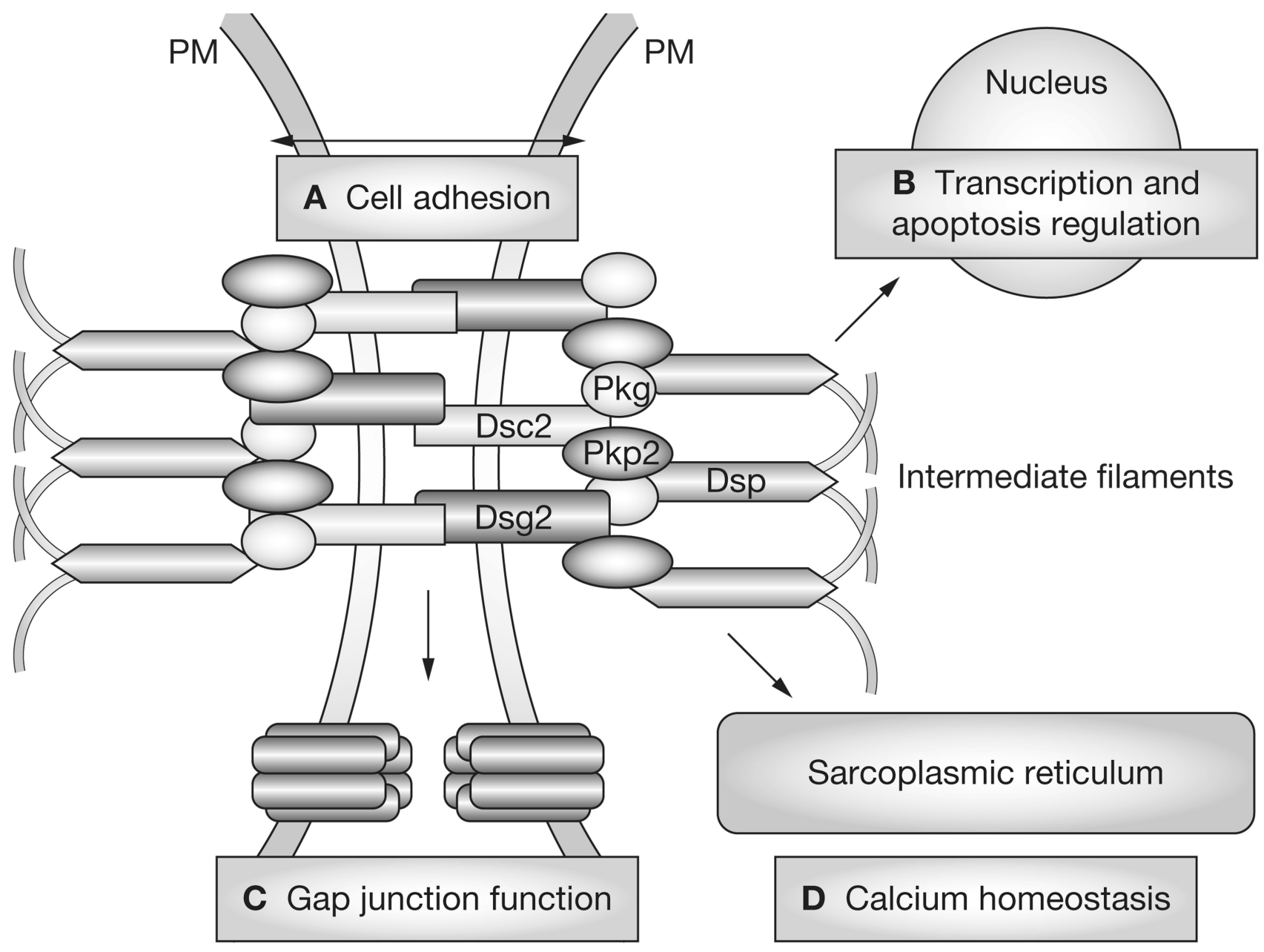

Figure 1.

The cardiac desmosome and proposed roles of the desmosome in (A) supporting structural stability through cell-cell adhesion, $(\mathbf{B})$ regulating transcription of genes involved in adipogenesis and apoptosis, and maintaining proper electrical conductivity through regulation of $(\mathbf{C})$ gap junctions and (D) calcium homeostasis. Abbreviations: Dsc2, desmocollin-2; Dsg2, desmoglein-2; Dsp, desmoplakin; Pkg, plakoglobin; Pkp2, plakophilin-2; PM, plasma membrane. 


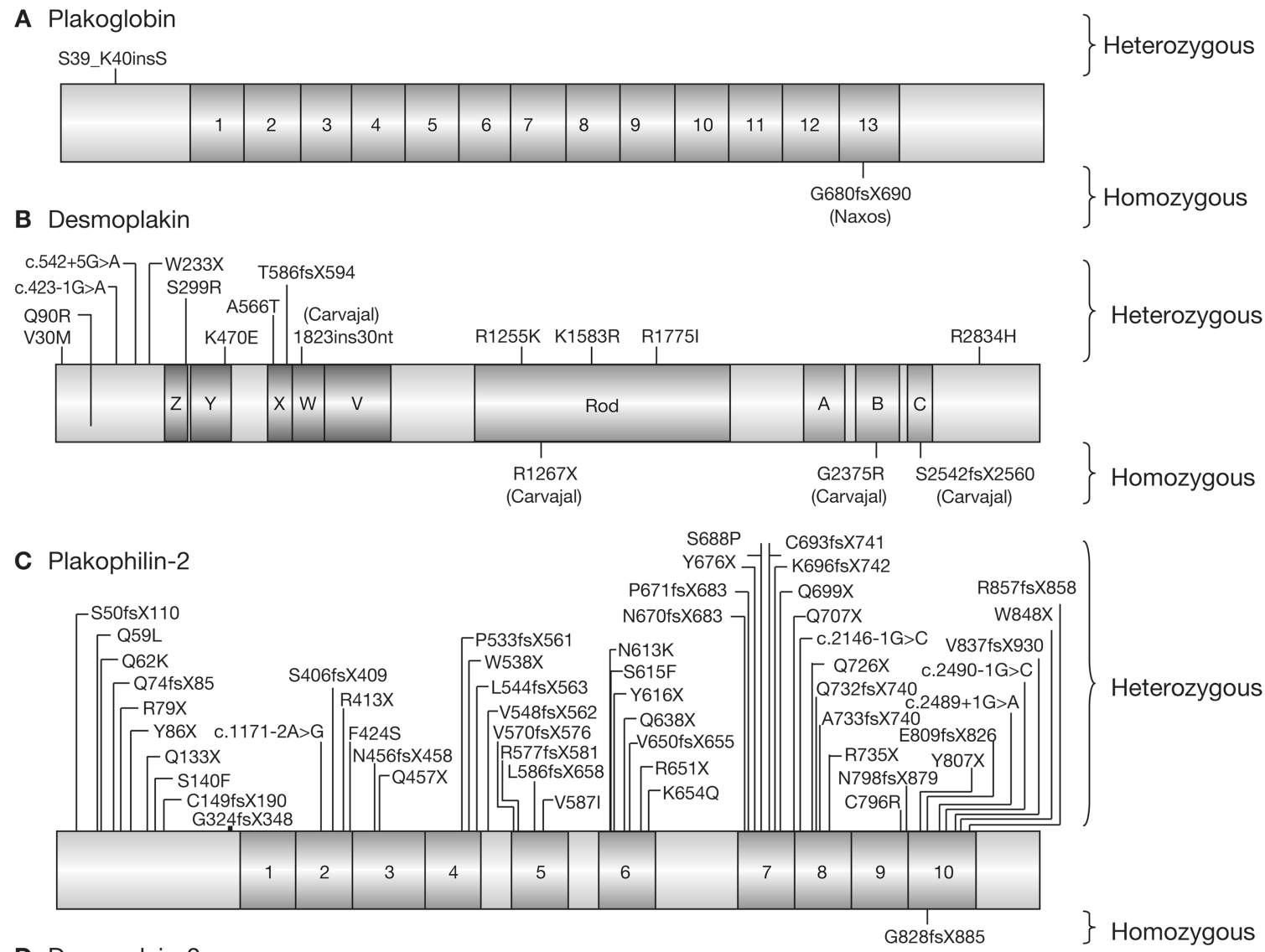

D Desmoglein-2

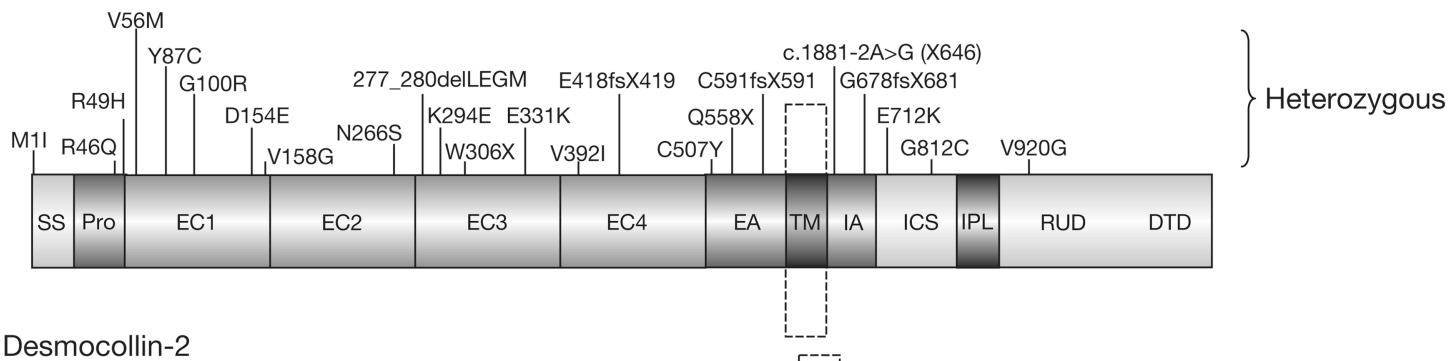

\section{E Desmocollin-2}

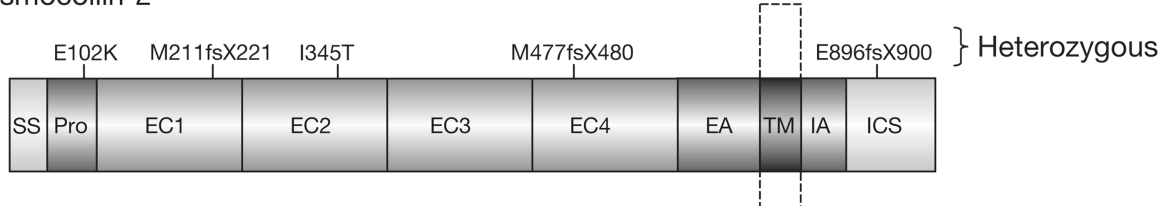

Figure 2.

Schematic of the five desmosomal proteins in which ARVD/C mutations have been identified and published. Heterozygous mutations are indicated above each diagram and homozygous mutations are indicated below each diagram. (A) Plakoglobin; the 13 armadillo domains are numbered. (B) Desmoplakin; the five $\mathrm{N}$-terminal $\alpha$-helical bundles $(\mathrm{Z}, \mathrm{Y}, \mathrm{X}, \mathrm{W}$, $\mathrm{V}$ ) are shown, followed by the rod domain required for dimerization and the $\mathrm{A}, \mathrm{B}$, and $\mathrm{C}$ subdomains of the plakin-repeat domain. (C) Plakophilin-2; the 10 armadillo domains are numbered. (D) Desmoglein-2 and (E) Desmocollin-2. Abbreviations: DTD, desmogleinspecific terminal domain; EA, extracellular anchor; EC1-4, extracellular domains 1-4; IA, intracellular anchor; ICS, intracellular cadherin segment; IPL, intracellular proline-rich 
linker; Pro, propeptide; RUD, 6 repeated-unit domains; SS, signal peptide sequence; TM, transmembrane domain. 


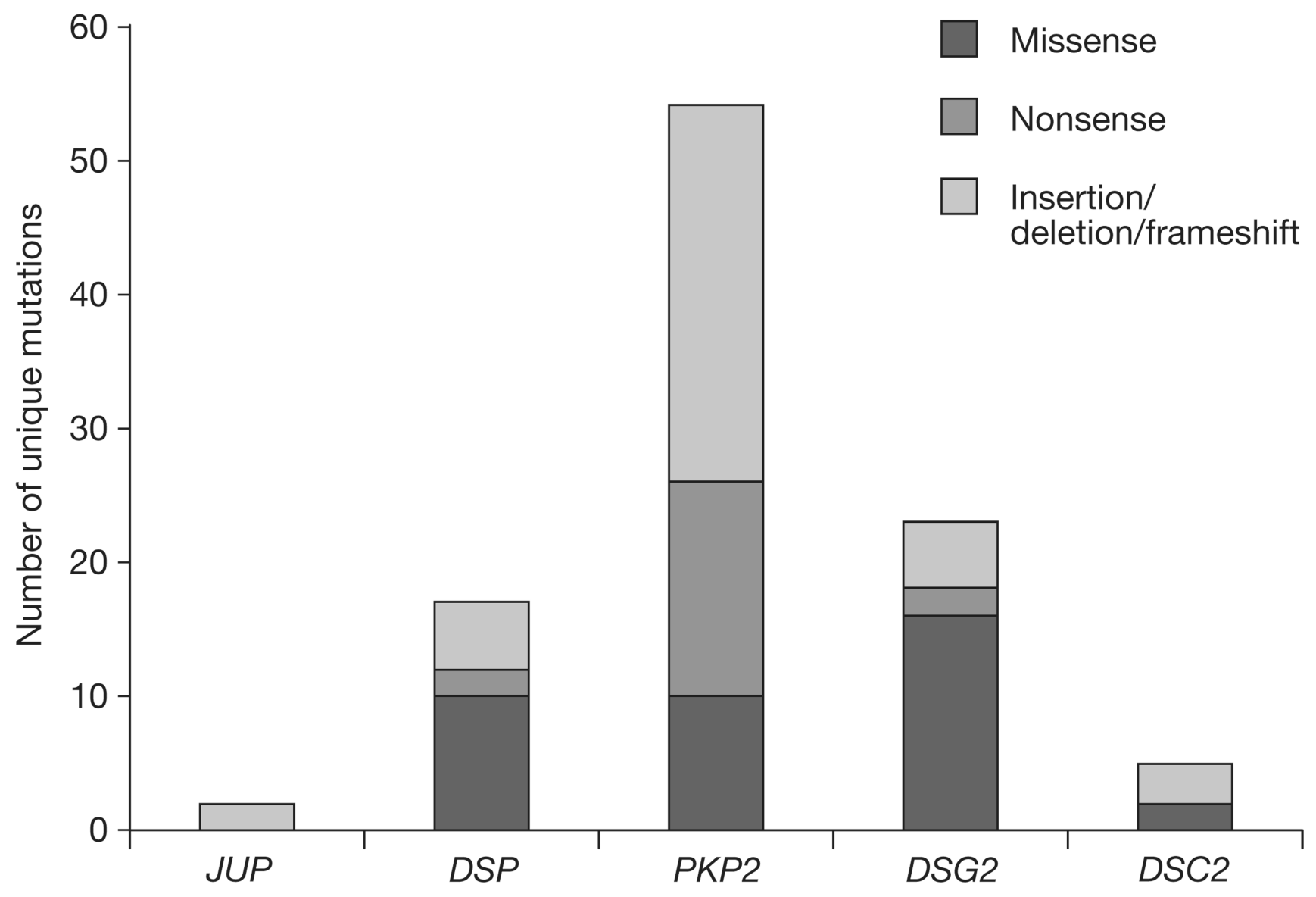

Figure 3.

Tallies of the types of unique mutations found in each of the five desmosomal genes mutated in arrhythmogenic right ventricular dysplasia/cardiomyopathy. Shown are missense, nonsense, and insertion/deletion or frameshift mutations. Abbreviations: DSC2, desmocollin-2; DSG2, desmoglein-2; DSP, desmoplakin; JUP, junctional plakoglobin; $P K P$ 2, plakophilin-2. 
Table 1

Arrhythmogenic right ventricular dysplasia/cardiomyopathy diagnostic criteria. To confirm diagnosis, individuals must fulfill two major criteria, or one major and two minor criteria, or four minor criteria, with each criterion coming from a different group.

\begin{tabular}{|c|c|c|}
\hline Group & Major criteria & Minor criteria \\
\hline $\begin{array}{l}\text { Structural or functional } \\
\text { RV abnormality }\end{array}$ & $\begin{array}{l}\text { Severe RV dilation and reduction of RV } \\
\text { ejection fraction with little or no LV involvement } \\
\text { Localized RV aneurysm } \\
\text { Severe segmental dilation of the right ventricle }\end{array}$ & $\begin{array}{l}\text { Mild global RV dilation and/or ejection fraction reduction } \\
\text { with normal LV } \\
\text { Mild segmental dilation of the right ventricle } \\
\text { Regional RV hypokinesia }\end{array}$ \\
\hline Tissue characterization & $\begin{array}{l}\text { Infiltration of RV myocardium by fibrofatty } \\
\text { replacement tissue }\end{array}$ & No criteria listed \\
\hline $\begin{array}{l}\text { Electrocardiogram } \\
\text { depolarization/ } \\
\text { conduction abnormality }\end{array}$ & $\begin{array}{l}\text { Epsilon waves or localized prolongation } \\
(>110 \mathrm{~ms}) \text { of the QRS complex in right } \\
\text { precordial leads (V1-V3) }\end{array}$ & Late potentials on signal-averaged electrocardiogram \\
\hline $\begin{array}{l}\text { Electrocardiogram } \\
\text { repolarization } \\
\text { abnormality }\end{array}$ & No criteria listed & $\begin{array}{l}\text { Inverted } \mathrm{T} \text { waves in electrocardiogram leads } \mathrm{V} 1-\mathrm{V} 3 \text {, aged } \\
>12 \text { years, without RBBB }\end{array}$ \\
\hline Arrhythmias & No criteria listed & $\begin{array}{l}\text { LBBB-type ventricular tachycardia (sustained or nonsustained) } \\
\text { Frequent premature ventricular contractions }(>1,000 \text { per } 24 \mathrm{~h})\end{array}$ \\
\hline Family history & $\begin{array}{l}\text { Family history of ARVD/C confirmed on } \\
\text { autopsy or surgery }\end{array}$ & $\begin{array}{l}\text { Family history of ARVD/C clinically and independently } \\
\text { diagnosed } \\
\text { Familial history of premature sudden death ( }<35 \text { years) owing to } \\
\text { suspected ARVD/C }\end{array}$ \\
\hline
\end{tabular}

Abbreviations: ARVD/C, arrhythmogenic right ventricular dysplasia/cardiomyopathy; LBBB, left bundle branch block; LV, left ventricular; RBBB, right bundle branch block; RV, right ventricular. Permission obtained from the BMJ Publishing Group 